Publ. RIMS, Kyoto Univ.

40 (2004), 883-903

\title{
Correspondence and Analyticity ${ }^{\dagger}$
}

By

\author{
Henry P. STAPP*
}

\begin{abstract}
The analyticity properties of the S-matrix in the physical region are determined by the correspondence principle, which asserts that the predictions of classical physics are generated by taking the classical limit of the predictions of quantum theory. The analyticity properties deducible in this way from classical properties include the locations of the singularity surfaces, the rules for analytic continuation around these singularity surfaces, and the analytic character (e.g., pole, logarithmic, etc.) of these singularities. These important properties of the S-matrix are thus derived without using stringent locality assumptions, or the Schroedinger equation for temporal evolution, except for freely moving particles. Sum-over-all-paths methods that emphasize paths of stationary action tend to produce the quantum analogs of the contributions from classical paths. These quantum analogs are derived directly from the associated classical properties by reverse engineering the correspondence-principle connection.
\end{abstract}

\section{$\S 1$. Introduction}

The S matrix was introduced by Wheeler [1]. It specifies the amplitude for the scattering of any set of originally noninteracting initial particles to any set of eventually noninteracting final particles. The $\mathrm{S}$ matrix is the aspect of relativistic quantum field theory most clearly related to empirical measurements.

The $\mathrm{S}$ matrix is a function of the momentum-energy four-vectors of the initial and final particles. The law of conservation of momentum-energy entails

Communicated by T. Kawai. Received October 29, 2003. Revised January 29, 2004.

2000 Mathematics Subject Classification(s): Primary 8102, 81U20; Secondary 46F15, $81 \mathrm{~T} 18$.

This work is supported in part by the Director, Office of Science, Office of High Energy and Nuclear Physics, Division of High Energy Physics, of the U.S. Department of Energy under Contract DE-AC03-76SF00098

${ }^{\dagger}$ This article is an invited contribution to a special issue of Publications of RIMS commemorating the fortieth anniversary of the founding of the Research Institute for Mathematical Sciences.

*Lawrence Berkeley National Laboratory, University of California, Berkeley, California 94720

(C) 2004 Research Institute for Mathematical Sciences, Kyoto University. All rights reserved. 
that the term of the $\mathrm{S}$ matrix that describes the scattering of any specified set of initial particles to any specified set of final particles must have a momentum-energy conservation-law delta function that constrains the sum of the momentum-energy vectors of the final particles to be equal to the sum of the momentum-energy vectors of the initial particles. The remaining factor, which is defined only at points that satisfy this conservation-law condition, is called a scattering function. It is finite at almost all points in its domain of definition. This is important because computations starting from the time-evolution equations tend to give scattering functions that are everywhere infinite. Thus Heisenberg [2] and others [3] have proposed an S-matrix approach to relativistic quantum theory that avoids the infinities that arise from the time-evolution equation by discarding that equation altogether, and computing the $\mathrm{S}$ matrix directly from certain of its general properties.

The pure S-matrix approach works well for simple cases. It may work in general, but new computational techniques would be needed to achieve this.

A key property of the scattering functions is that each of them is analytic (holomorphic) at almost every point of its original (real) domain of definition. This property was originally deduced from an examination of Feynman's formulas for these functions, which are derived essentially from the time-evolution equations. Landau [4] and Nakanishi [5] independently deduced the very restrictive necessary conditions for the occurrence of singularities of these functions. Coleman and Norton [6] then noted that these Landau-Nakanishi conditions are precisely the conditions for the existence of a classical physical process that has the same topological structure - i.e., has the same arrangement of line segments connected at vertices — as the Feynman graph with which it is associated.

A Feynman graph is topological structure of line segments joined at vertices. It was used by Feynman to specify a corresponding mathematical contribution to the $\mathrm{S}$ matrix. The associated Landau-Nakanishi diagram is a diagram in four-dimensional space-time that has the same topological structure, but moreover satisfies all of the conditions of a corresponding process in classical physics. Thus a Landua-Nakanishi diagram can be regarded as a representation of a process in classical-physics that consists of a network of point particles that interact only at point vertices, and that propagate between these vertices as freely moving particles.

The rules of (relativistic) classical particle physics assign a momentumenergy four-vector to each line of the diagram, and impose the conservation-law condition that the energy-momentum flowing into the diagram along the initial 
incoming lines must be able to flow along the lines of the graph, and then out along the final outgoing lines with energy-momentum conserved at each vertex. This conservation-law condition is imposed also by the Feynman rules. But the Landau-Nakanishi (i.e., classical-physics) diagram is required to satisfy also the "classical physics" requirement that each line of the spacetime diagram be a straight-line segment that is parallel to the momentum-energy carried by that line. [In classical relativistic particle physics each freely-moving particle moves in space-time in the direction of its momentum-energy four-vector $\left(p=m v, v^{2}=1\right)$, but this property is not imposed in quantum theory: it would conflict with the uncertainty principle, and, likewise, with the Fouriertransformation connection between space-time displacements and momentumenergy that constitutes the foundation of quantum theory.]

The Landau-Nakanishi diagram is, then, the picture of a possible classical process, involving point particles interacting at points, and conforming to the conditions of relativistic classical-particle physics. These conditions were shown by Landau and Nakanishi to specify the location (in the space of the momentumenergy four vectors of the initial and final particles) of a singularity-failure of analyticity - of the contribution to the $S$ matrix corresponding to the associated Feynman graph.

The purpose of this article is to highlight the fact that although this important connection between the physical-region singularities of the quantum scattering functions and associated classical scattering processes was originally derived from very strong quantum assumptions involving the concepts of point interactions and continuous Schroedinger evolution in time, the result is actually a consequence of much less. It is a consequence of the "correspondence principle" connection between relativistic quantum physics and relativistic classicalparticle physics. This principle asserts that the predictions of classical physics emerge from quantum theory in the "classical limit" in which all effects due to the nonzero value of Planck's constant become negligible.

The correspondence principle entails, however, much more than just the analyticity of the $\mathrm{S}$ matrix at all points that do not correspond to a classicalphysics process. It entails also that, in a real neighborhood of almost every real singular point, the scattering function is the limit of a function analytic in the interior of a certain cone-like domain that extends some finite distance into the complex domain from its tip in the real neighborhood. This means that each physical scattering function is a limit of single analytic function. That feature of the $\mathrm{S}$ matrix is one of the key general properties upon which the $\mathrm{S}$-matrix approach is based. Its derivation from the correspondence principle 
was given by Chandler and Stapp [7] and by Iagolnitzer and Stapp [8]. The first of these two papers sets out the general framework, but is formulated within a distribution-analytic framework in which the wave functions are, apart from mass-shell-constraint delta functions, infinitely differentiable functions of compact support. Consequently, it achieves analyticity only modulo infinitely differentiable background terms. The second of these papers uses essentially Gaussian wave functions to obtain full analyticity.

It is worth noting that Sato [9] independently constructed a mathematical machinery called the sheaf of microfunctions, which can be used to describe the same cone-like domain when applied to the $\mathrm{S}$ matrix.

The correspondence principle entails even more. It specifies also the $n a$ ture of these singularities: whether they are, for example, pole, or logarithmic singularities. This means that the quantum effects closely associated with these classical-physics processes are determined already by the correspondence principle, without appeal to the notion of true point interactions or of the relativistic generalization of the Schroedinger equation. That is, the correspondence principle, which is a condition on the classical limit of quantum theory, can be "reverse engineered" to deduce those features of the quantum S matrix that produce the classical result in the classical limit. And these feature include the analytic character of the $\mathrm{S}$ Matrix scattering functions in their original (real) domains of definition.

\section{§2. An Asymptotic Fall-Off Property}

The papers with Chandler and Iagolnitzer just cited deal exclusively with particles of non-zero rest mass. The momentum-space wave function of particle $i$ then has, due to the mass-shell condition, the form

$$
\Psi_{i}\left(p_{i}\right)=\psi_{i}\left(p_{i}\right) 2 \pi \delta\left(p_{i}^{2}-m_{i}^{2}\right),
$$

where $p_{i}^{2}$ is the Minkowsky inner product of $p_{i}$ with itself, with metric $(1,-1$, $-1,-1$ ), and $m_{i}$ is the (nonzero) rest-mass of particle $i$. Quantum theory is characterized, fundamentally, by the Fourier-transform link between momentum-energy and space-time. Thus the spacetime form of this momentum-energy wave function is given by the Fourier transform:

$$
\widetilde{\Psi}_{i}\left(x_{i}\right)=\int(2 \pi)^{-4} d^{4} p_{i} \exp \left(-i p_{i} x_{i}\right) \Psi_{i}\left(p_{i}\right) .
$$

The spacetime wave function has important asymptotic fall-off properties. In Appendix A of reference [19] it is shown that if $\psi_{i}\left(p_{i}\right)$ has compact support 
and is continuous, together with its first and second derivatives, and if $v$ is any positive time-like four-vector satisfying $v^{2}=1$, then

$$
\lim _{\tau \rightarrow \infty} f\left(m_{i}, \tau\right) \widetilde{\Psi}_{i}(v \tau)=\psi_{i}\left(m_{i} v\right),
$$

where

$$
f\left(m_{i}, \tau\right)=2 m_{i}\left(2 \pi i \tau / m_{i}\right)^{2 / 3} \exp \left(i m_{i} \tau\right) .
$$

In the formula (2.2) the expression $p_{i} x_{i}$ in the exponent is originally divided by Planck's constant over $2 \pi$. But that factor has been removed by choosing units of space and time so that Planck's constant (divided by $2 \pi$ ) and the velocity of light are both unity. But then letting $\tau$ go to infinity is effectively equivalent to letting Planck's constant go to zero: the expansion of the spacetime scale is mathematically equivalent to going to the classical limit. Formula (2.3) shows that in this limit the probability distribution in spacetime for a freely moving particle is specified by the momentum-space distribution function $\psi_{i}\left(p_{i}\right)$ in accordance with the relativistic classical physics formula $p_{i}=m_{i} v$.

The fall-off property described above was derived from quantum theory. Later I shall derive it from classical physics.

The correspondence principle asserts that the classical-physics results hold not only for these free-particle states but also for processes corresponding to networks of locally interacting particles that propagate freely over the asymptotically large distances between their interactions: the classical physics probabilities emerges from the quantum probabilities in the asymptotic $\tau \rightarrow \infty$ limit. This correspondence-principle requirement determines not only the locations and natures of the singularities of the quantum momentum-space scattering functions, but normally entails also that, in a real neighborhood of a singular point $P$, the scattering function is a limit of a function analytic in the intersection of a complex neighborhood of $P$ with the interior of a cone that extends from the real domain in a set of directions that is specified by the structures of the classical scattering diagrams associated with that singular point $P$. This connection between directions of analyticity at singularities and classical spacetime diagrams is made via a $4 n$-dimensional displacement vector $U$ introduced in reference [7].

\section{§3. The $4 n$-dimensional Displacement Vector $U$}

Consider a spacetime diagram $D$ that describes a possible network of classical particles with a total of $n$ initial and final particles. This diagram $D$ 
determines (via the directions of the initial and final lines) a set $P=\left(p_{1}, \ldots, p_{n}\right)$ of initial and final momentum-energy vectors.

It is convenient to introduce in addition to the physical momentum-energy vectors $p_{i}$, which have positive energy components, also the mathematical momentum-energy vectors $k_{i}$, where $k_{i}=p_{i}$ for initial particles, and $k_{i}=-p_{i}$ for final particles. Then the law of conservation of energy momentum reads $\sum k_{i}=0$.

The $4 n$-dimensional displacement vector $U$ is defined as follows. From any arbitrarily chosen origin $O$ in spacetime draw, for each initial and final particle $i$, a vector $u_{i}$ from $O$ to some point on the straight-line that contains the initial or final line $i$. Define

$$
U=\left(u_{1}, \ldots, u_{n}\right)
$$

For a fixed spacetime diagram $\mathrm{D}$ this $4 \mathrm{n}$-dimensional displacement vector $U$ is not uniquely fixed: one can add to $U$ any vector of the form

$$
U_{0}=\left(a+b_{1} k_{1}, a+b_{2} k_{2}, \ldots, a+b_{n} k_{n}\right),
$$

where a is a real spacetime vector, and for each $i$ the parameter $b_{i}$ is a real number. Changing $a$ just shifts the location of $D$ relative to the origin $O$, and changing $b_{i}$ just slides the tip of $u_{i}$ along the straight line $i$.

Notice that the combination of the four conservation-law delta functions and the $n$ mass-shell delta functions restricts the relevant set of points in the $4 n$-dimensional space of points $K=\left(k_{1}, \ldots, k_{n}\right)$ to a surface of co-dimension $4+n$, and that the $4+n$ dimensional set of vectors $U_{0}$ spans the set of normals to that co-dimension $4+n$ surface: the contravariant vectors formed by taking linear combinations of the gradients to the arguments of the $4+n$ delta functions constitute the set of vectors $U_{0}$. This is the simplest example of the important fact that the set of vectors $U$ associated with a singular point $K$ generally span the space defined by the set of normal vectors to the surface of singular points passing though $K$. This normality of the vectors $U$ associated with diagrams of classical physics to the surfaces of singularities of the $\mathrm{S}$ matrix provides the link between relativistic classical physics and domains of analyticty of scattering functions in relativistic quantum physics.

\section{$\S 4$. Another Asymptotic Fall-Off Property}

If the wave function $\psi_{i}\left(p_{i}\right)$ in Eq. (2.1) is infinitely differentiable and of compact support, and if $V$ is the associated velocity (double) cone consisting 
of all lines through the origin $\left(p_{i}=0\right)$ that intersect the compact support (in the mass shell $\left.p_{i}^{2}=m_{i}^{2}\right)$ of $\psi_{i}\left(p_{i}\right)$ then, for all $u$ in any compact set that does not intersect $V$, the function $\widetilde{\Psi}(u \tau)$ uniformly approaches zero faster than any inverse power of the scale parameter $\tau$ : for any integer $N$

$$
\lim _{\tau \rightarrow \infty} \tau^{N} \widetilde{\Psi}_{i}(u \tau)=0 .
$$

This is a standard result (cf. ref [8], Eqn. (28)), and it allows one to prove the weaker analyticity properties that hold modulo infinitely differential background terms. (See ref. [7]). But to derive full analyticity from the correspondence principle a stronger fall-off property is needed.

This stronger asymptotic fall-off property is obtained by introducing into the wave functions $\psi_{i}\left(p_{i}\right)$ an exponential factor that shrinks in width as $\tau$ tends to infinity. Specifically, one introduces free-particle momentum-space wave functions of the form

$$
\psi_{\tau, \gamma, \bar{p}}(p)=\chi(p) \exp \left(-(p-\bar{p})^{2} \gamma \tau\right)
$$

and also requires the infinitely differential function $\chi(p)$ (of compact support) to be analytic at $p=\bar{p}$, where $p^{2}=\bar{p}^{2}=m^{2}$. Then the following fall-off property holds: for all 4 -vectors $u$ in any compact set that does not intersect the line through the origin containing $\bar{p}$, and for all $\gamma \geq 0$ smaller than some fixed $\gamma_{0}$, there is a pair of finite numbers $(C, \alpha)$ such that for all $\tau$

$$
\left|\widetilde{\Psi}_{\tau, \gamma, \bar{p}}(u \tau)\right|<C \exp -\alpha \gamma \tau \text {. }
$$

Classical and quantum proofs of this fall-off property will be described below. But let us first show how this property of the free-particle coordinatespace wave functions is used to deduce, from the correspondence principle, domains of analyticity for the momentum-space scattering function.

\section{$\S 5 . \quad$ Kinematics and Probabilities}

The connection to the correspondence principle is obtained by using initial and final wave functions $\Psi_{i}\left(p_{i}, u_{i}\right)$ of the form

$$
\Psi_{i}\left(\tau, \gamma, \bar{p}_{i} ; p_{i}, u_{i}\right)=\Psi_{i}\left(\tau, \gamma, \bar{p}_{i} ; p_{i}\right) \exp i u_{i} p_{i}
$$

where, for any $i$, in accordance with (2.1) and (4.2),

$$
\Psi(\tau, \gamma, \bar{p} ; p)=\psi_{\tau, \gamma, \bar{p}}(p) 2 \pi \delta\left(p^{2}-m^{2}\right)
$$


The wave function (5.1) represents the particle state obtained by translating the state represented by $\Psi_{i}$ by the spacetime displacement $u_{i}$. The parameters $\gamma$ are taken to be the same for all $i$. It is convenient to use henceforth real $\chi_{i}\left(p_{i}\right)$, each of which is equal to one (unity) in some finite neighborhood of $\bar{p}_{i}$.

The correspondence-principle results are obtained by examining the $\tau \rightarrow$ $\infty$ behaviour of the transition amplitude

$$
A(\tau)=S\left[\left\{\Psi_{i}\left(\tau, \gamma, \bar{p}_{i} ; p_{i}, u_{i} \tau\right)\right\}\right]
$$

where the right-hand side is

$$
\left[\prod_{i} \int(2 \pi)^{-4} d^{4} k_{i} \Psi_{i}\left(\tau, \gamma, \bar{k}_{i} ; k_{i}\right)\right] S(K) \exp i K U \tau .
$$

The absolute value squared of the complex number $A(\tau)$, times $f(\tau)$, is the transition probability associated with these states of the initial and final particles, and $f(\tau)$ is the inverse of the square of the product of the norms of the wave functions $\psi_{i}$ of (4.2). This factor grows like $(\tau)^{3 n}$, but this growth can be absorbed into a bound of the form $C \exp -\alpha \gamma \tau$ by a slight adjustment of $C$ and $\alpha$.

\section{$\S 6 . \quad$ The Correspondence-Principle Condition}

For any fixed $\bar{K}$ (with $\sum \bar{k}_{i}=0$ and, for each $i, \bar{k}_{i}^{2}=m_{i}^{2}$ ) there is a set $C(\bar{K})$ of vectors $U$ such that each pair of $4 n$-dimensional vectors $(\bar{K}, U)$ satisfies the Landau-Nakanishi conditions. This set $C(\bar{K})$ includes the set $C_{0}(\bar{K})$ consisting of all of the vectors $U_{0}$ of the form (3.2): each of these vectors $U_{0}$ specifies a classical-physics diagram $D$ in which all of the initial and final particles pass through a single common point. Each of these vectors $U_{0}$ has a null (Lorentz) inner product with every tangent vector to - i.e., with every infinitesimal displacement in - the surface at $\bar{K}$ of singularities generated by the mass-shell and overall conservation-law delta functions.

Suppose $C(\bar{K})=C_{0}(\bar{K})$. That would mean that, on the one hand, there are for the set $\left\{\bar{k}_{i}\right\}$ of initial and final (mathematical) momentum-energy vectors specified by $\bar{K}$ no classical-physics diagrams except the trivial ones in which all the initial and final particles pass through a common point, and, on the other hand, according to the Feynman rules, no singularity of the quantum scattering function. But from the S-matrix point of view the Feynmam rules are suspect, because they come essentially from the physically meaningless continuous time evolution, and also lead to infinities. However, the general correspondence principle condition that the predictions of classical physics should emerge in the 
limit where Planck's constant goes to zero, or, equivalently, where $\tau$ goes to infinity, would seem to be an exceedingly plausible and secure condition. The analyticty of the scattering function at this point $\bar{K}$ is, in fact, a consequence of that correspondence condition.

For any point $\bar{K}$ such that $C(\bar{K})=C_{0}(\bar{K})$ consider any $U$ that does not belong $C(\bar{K})$. If $U$ does not belong to $C(\bar{K})=C_{0}(\bar{K})$ then for at least one of the $n$ particles $i$ the component vector $U_{i}$ is not parallel to $\bar{k}_{i}$. But then the amplitude $A(\tau)$ will pick up an exponential fall-off factor of the kind shown in (4.3). These vectors $U$ cover a unit sphere in the $3 n-4$-dimensional subspace that is normal to the $n+4$-dimentional subspace $C(\bar{K})$. Thus there will be a least value of $\alpha$ for the $U$ 's on this (compact) unit sphere.

This uniform exponential fall-off over this unit sphere arises, in the classical computation, from the exponential fall off of the overlap of the probability functions of the initial and final particles: i.e., from the exponentially decreasing probability, as $\tau$ increases, for all of the initial and final particles to be in any single finite region of space-time that grows like the square root of $\tau$. In classical physics such an exponential decrease in this probability, coupled with the fact that the only classical scattering process that can carry the initial momentum-energies to the final momentum-energies is one where all the initial and final particle trajectories pass through some such growing space-time region entails a similar fall off of the transition probabilities: the probability for this kind of classical process to occur cannot grow faster than the product of the probabilities that the particle can all be in any such growing region. Thus the correspondence principle requires that transition amplitude $A(\tau)$ have the same sort of fall off as the one arising from the overlap of the wave functions. It will now be shown that this condition entails the analyticity of the scattering function at this point $\bar{K}$ where $C(\bar{K})=C_{0}(\bar{K})$.

\section{$\S 7 . \quad$ Derivation of Analyticity at Trivial Points}

By a "trivial point" I mean a point $\bar{K}$ such that $C(\bar{K})=C_{0}(\bar{K})$ : the only classical processes with external momenta specified by $\bar{K}$ are the trivial single-vertex diagrams.

The set of Landau-Nakanishi surfaces that enter any bounded region of $K$ space has been shown to be finite [Ref. 10]. And each such surface is confined to a co-dimension-one analytic manifold. Consequently, each trivial point $\bar{K}$ lies in an open neighborhood of such points.

Introduce a set of analytic coordinates $q$ in the $3 n$-4-dimensional manifold in $K$-space restricted by the mass-shell and conservation-law conditions near 
$\bar{K}$. Let the $q$ be a subset of the space components of the set of vectors $\left(k_{i}-\bar{k}_{i}\right)$, and let the $v$ associated with any $q(K)$ in the neighborhood of $q(\bar{K})=0$ be the corresponding $3 n-4$ components of $U \tau \bmod C_{0}(K)$, so that $K U \tau$ in (5.2) becomes $(-q v-\bar{k} v)$, where the metric $(1,1,1)$ is now used, and $v$ represents displacements away from the displacements that generate the trivial singlevertex processes. Then the $A(\tau)$ in (5.2), times the (unimportant) phase factor $\exp (i \bar{k} v)$. can be written as

$$
T(v, r)=\int d q F(q) \exp (-r \mu(q)) \exp (-i q v),
$$

where

$$
\mu(q)=\sum_{i}\left(k_{i}(q)-k_{i}(0)\right)^{2},
$$

$r=\gamma \tau$, and $F(q)$ is the scattering function times a factor that is real, infinitely differentiable of compact support, and analytic at $q=0$, which is the $q$-space image of $\bar{K}$. A fall-off property of the form (4.3) is required to hold for all $\tau$ and all $0 \leq \gamma \leq \gamma_{0}$, with $r=\gamma \tau$, and all $v=\hat{v} \tau$ with $|\hat{v}|=1$. What needs to be proved is that this fall-off condition, together with the analogous rapid (faster than any power of $\tau$ ) fall off at $\gamma=0$, entails the analyticity of $F(q)$ at $q=0$.

This rapid fall off of the bounded $T(v, 0)=T(\hat{v} \tau, 0)$ for all unit vectors $\hat{v}$ means that $F(q)$ is the well-defined and infinitely differentiable Fourier transform:

$$
F(q)=(2 \pi)^{l} \int d v \exp (i q v) T(v, 0)
$$

where $l=3 n-4$. To show that $F(q)$ is analytic at $q=0$ re-write this equation in the form

$$
\begin{aligned}
& (2 \pi)^{l} F(q)=\int d v \exp (i q v) \times \\
& {\left[T\left(v, \gamma_{0}|v|\right) \exp \left(\gamma_{0}|v| \mu(q)\right)-\int_{0}^{\gamma_{0}|v|} d r \frac{\partial}{\partial r}[T(v, r) \exp (r \mu(q))]\right] .}
\end{aligned}
$$

Consider first the first term in the big brackets. The correspondence principle requires the factor $T\left(v, \gamma_{0}|v|\right)$ to be bounded by $C \exp \left(-\alpha \gamma_{0}|v|\right)$. The function $\mu(q)$ is zero at $q=0$, and hence the associated exponential growth is dominated by the fall-off factor for $q$ in a sufficiently small neighborhood of $q=0$. Indeed, this bound keeps the integral well defined and analytic for all 
$q$ in a small complex neighborhood of $q=0$. Thus the contribution $F_{1}(q)$ to $F(q)$ coming from the first term in the big brackets is analytic at $q=0$.

To prove that this property holds also for the other contribution, $F_{2}(q)$, substitute (7.1) into the second term in the big brackets. The $\partial / \partial r$ can be moved under the integral over $d q$ because $F(q)$ is infinitely differentiable of compact support. This gives for the integrand

$$
\begin{aligned}
& \exp (i q v) \frac{\partial}{\partial r}[T(v, r) \exp (r \mu(q))] \\
& \quad=\int d q^{\prime} F\left(q^{\prime}\right) \exp \left(i\left(q-q^{\prime}\right) v\right) \exp \left(r\left(\mu(q)-\mu\left(q^{\prime}\right)\right)\right)\left[\mu(q)-\mu\left(q^{\prime}\right)\right]
\end{aligned}
$$

Hefer's theorem [8] allows one to write

$$
\mu(q)-\mu\left(q^{\prime}\right)=\rho\left(q, q^{\prime}\right) \cdot\left(q-q^{\prime}\right)
$$

where $\rho$ is a vector whose the components $\rho_{j}(\mathrm{j}=1, \ldots, 3 \mathrm{n}-4)$ are analytic in a product of domains around $q=0$, and $q^{\prime}=0$. Then (7.5) becomes

$$
\exp (i q v) \frac{\partial}{\partial r}[T(v, r) \exp (r \mu(q))]=D i v_{v}[\exp (i q v) \exp (r \mu(q)) H(q, v, r)],
$$

where $H(q, v, r)$ is the vector

$$
H(q, v, r)=-i \int d q^{\prime} F\left(q^{\prime}\right) \exp \left(-i q^{\prime} v\right) \exp \left(-r \mu\left(q^{\prime}\right)\right) \rho\left(q, q^{\prime}\right) .
$$

We may thus write

$$
(2 \pi)^{l} F_{2}(q)=-\lim _{R \rightarrow \infty}\left[\int_{|v|<R} d v \int_{0}^{\gamma_{0}|v|} d r \operatorname{Div} v[\exp (i q v) \exp (r \mu(q)) H(q, v, r)]\right] .
$$

For fixed $R$ we can change the order of integration and perform first an integration over $v$ for $r / \gamma_{0}<|v|<R$. Then Gauss' theorem gives the volume integral of the divergence as the difference of two surface integrals, one at $|v|=r / \gamma_{0}$, the other at $|v|=R$. The estimates given in Appendix IV of ref. [10] show that the contribution at $R$ vanishes as $R \rightarrow \infty$. The contribution at $|v|=r / \gamma_{0}$ integrated on $r$ from 0 to $\infty$ generates an integration over all $v$ with $r$ replaced by $|v| \gamma_{0}$, and a Jacobian factor $J(q)$ that is analytic at $q=0$. Thus we obtain

$$
(2 \pi)^{l} F_{2}(q)=\gamma_{0} \int d v \exp (i q v) \exp \left(\gamma_{0}|v| \mu(q)\right) \widehat{v} \cdot H\left(q, v, \gamma_{0}|v|\right),
$$


where $\widehat{v}=v /|v|$. This function $F_{2}(q)$ is analytic at $q=0$ for the same reasons that $F_{1}(q)$ was. This completes the proof, apart from the straightforward calculations given in Appendix IV of reference [10].

Note that (7.1), with $r=\gamma_{0} \tau$, and (7.4) together with (7.10), gives a generalization of the Fourier transformation theorem that incorporates Gaussian factors. It gives, from the mathematical point of view, a localized version of the familiar connection between analyticity and exponential fall off of the Fourier transform. From the physics point of view it gives a connection between the analyticity of the scattering functions of relativistic quantum theory and the results of classical physics that emerge from quantum theory in the classical limit where Planck's constant goes to zero.

The analyticity of the scattering functions except on the Landau-Nakanishi surfaces has thus been derived, by "reverse engineering" the correspondence principle: quantum properties have been deduced from classical properties, the correspondence principle, and the basic connection between classical and quantum physics, namely the Fourier-transform connection between the momentumenergy and the space-time displacements of freely moving particles.

\section{$\S 8$. Derivation of Cone of Analyticity at Most Singular Points}

A more complex category of points $\bar{K}$ consists of points $\bar{K}$ such that all of the spacetime diagrams corresponding to this $\bar{K}$ are the same apart from shifts in location or scale, but which differ from the simple single-vertex case except in the limit where the diagram is shrunk to a point. For any such point $\bar{K}$ the set $C(\bar{K})$ consists of $C_{0}(\bar{K})$ plus a single ray, $U(\bar{K})$ : the displacements along $U(\bar{K})$ generate the displacements of the external lines of the diagram away from positions where they all intersect at a single point. [The argument can be extended to cover all points $\bar{K}$ such that all of the Landau-Nakanishi surfaces that contain $\bar{K}$ coincide with a single co-dimension-one Landau-Nakanishi surface, and hence all specify the same unique ray $U(\bar{K})$.]

It is important that $U(\bar{K})$ is a ray, not a full line: a displacement in the opposite direction does not give the locations of the external lines of a classically allowed process. (The intermediate particles would have to move backward in time, and carry the incoming positive energy backward in time.) Thus a compact set of displacements $U$ not in $C(\bar{K})$, but confined to a space essentially normal to the set $C_{0}(\bar{K})$, cannot now cover an entire sphere: there must be a hole in this compact set through which the single ray $U(\bar{K})$ can pass. 
To deal with this case one can introduce the same set of local coordinates $(q, v)$ as before, with $\hat{v}=v /|v|$, and let $\hat{v}(\bar{K})$ be the point on the unit sphere $|v|=1$ that is the image in $|v|=1$ of $U(\bar{K})$. Let $A(\bar{K})$ be a compact set in $v$ space that lies in the unit sphere, and covers this sphere $|v|=1$ except for points in a small open spherical ball about the point $\hat{v}(\bar{K})$. Let the points in this ball that lie also on the sphere $|v|=1$ be called $H(\bar{K})$ (for Hole), so that each point on $|v|=1$ lies either in $A(\bar{K})$ or in $H(\bar{K})$, but not in both.

Choose the functions $\chi\left(p_{i}\right)$ in (4.2) so that their supports are small enough so that the point $\hat{v}(K)$ corresponding to each point $K$ in the support of the product of the $\chi\left(p_{i}\right)$ s lies in a closed subset of the open set $H(\bar{K})$. Then for all points $\hat{v}=v /|v|$ in $A(\bar{K})$ the function $T(v, \gamma|v|)$ will, by virtue of the correspondence principle, fall off faster than any power of $|v|$ for $\gamma=0$, and like (4.3) for $0<\gamma \leq \gamma_{0}$. The problem is then to show that the function $F(q)$ in (7.1) is the boundary value, in some real neighborhood of $q=0$, of a function analytic in the intersection of a complex neighborhood of $q=0$ with an open cone $Q$ in $\operatorname{Im} q$.

To prove this, separate the $v$-space domain of integration in (7.3) into two disjoint parts, $V(H(\bar{K}))$ and $V(A(\bar{K}))$, where the latter consists of all rays from $v=0$ that pass through the closed set $A(\bar{K})$ of points in the sphere $|v|=1$, and $V(H(\bar{K}))$ is the rest of $v$ space.

This separation of the space of integration of the (bounded-by-virtue-ofunitarity) function $T(v, 0)$ into two parts separates $F(q)$ into two terms:

$$
F(q)=F_{H}(q)+F_{A}(q) .
$$

The imaginary part of $q$ in $F_{H}(q)$ is restricted to the open cone $Q$ in which $I m$ $q v>0$ for all $v$ in a closed cone $V$ that contains the closure of $V(H(\bar{K}))$ in its interior, apart from the origin $v=0$. For these $q$ the exponential factor exp $i q v$ in (7.3) get from $\operatorname{Im} q$ a factor $\exp -\alpha|\operatorname{Im} q||v|$, where $\alpha>$ const $>0$. This means, because $T(v, 0)$ is bounded, that the integral is absolutely convergent, and hence that $F_{H}(q)$ is analytic near $q=0$ for $\operatorname{Im} q$ in $Q$.

Most of the real points $q$ very near to $q=0$ are "trivial" points, of the kind considered in the preceding section. At those trivial points $q^{\prime}$, the function $F\left(q^{\prime}\right)=F_{H}\left(q^{\prime}\right)+F_{A}\left(q^{\prime}\right)$ is analytic. These two terms are taken at these points $q^{\prime}$ to be just the contributions to $F(q)$ specified in (7.4) and (7.10) restricted to the regions $V(H(\bar{K}))$ and $V(A(\bar{K}))$ respectively. Both of these contributions are analytic in the intersection of some neighborhood of $q$ with the cone $Q$. Thus one can stay in the domain of analyticity by moving $\operatorname{Im} q$ slightly into the cone $Q$ in order to pass to the other side of the surface of singularities that passes through $q=0$. 
A more elaborate presentation of this argument, and of its generalizations to more complex cases, can be found in references [7] and [8], and also in Iagolnitzer's book [11].

\section{§9. Correspondence-Principle Asymptotic Fall Off}

I have described some of the analytic consequences of the fall-off properties (2.3), (4.1), and (4.3). I turn now to a fuller discussion of the roots of these fall-off properties in the correspondence to classical properties.

The statistical predictions of quantum mechanics correspond, at least in a formal way, to the predictions of classical statistical mechanics. In the latter theory one describes a system of $n$ particles at any time $t$ in terms of a function $\rho(x, p, t)$, which specifies how the probability is distributed over the points $(x, p)$ of "phase space," where $x$ specifies the $3 n$ coordinate variables and $p$ specifies the $3 n$ momentum-space variables. Free-particle evolution keeps $p$ fixed and shifts the location $x_{i}$ of a particle of (rest) mass $m_{i}$ during a time interval $t$ to the location $x_{i}+t p_{i} / m_{i}$. For large $t$ the second term dominates, and the coordinate-space probability function goes over to the momentum-space probability function, properly scaled to account for the diverging directions of the different momentum vectors. This classical kinematics entails that for free particles the classical distribution $\rho(x, p, t)$ at large times $t$ becomes a product over $i$ of functions

$$
\rho\left(u_{i} t, p_{i}, t\right)=\left|\rho\left(u_{i} m_{i}\right) \rho\left(p_{i}\right) / f\left(m_{i}, t\right)^{2}\right|,
$$

where

$$
\rho\left(p_{i}\right)=\int d^{3} x_{i} \rho\left(x_{i}, p_{i}, t^{\prime}\right),
$$

is independent of $t^{\prime}$, and $f\left(m_{i}, t\right)$ is the function defined in (2.4). Here I am, for simplicity, assuming that the momenta are small enough so that the nonrelativistic formulas (where $t=\tau$ and $p_{0}=m$ ) are adequate. (The fully covariant formulation gives the same results.) The factor $\left(m_{i} / t\right)^{3}$ coming from $f\left(m_{i}, t\right)^{-2}$ compensates for the linear spreading out of the probability distribution in coordinate space, and the $1 /\left(2 m_{i}\right)^{2}$ comes from the normalization in (2.1). This equality of the classically-derived and quantum-mechanically derived limits constitutes, in this case, part of the correspondence-principle relationship between the asymptotic properties in classical and quantum theory: both theories give the same asymptotic form for the probability distribution in $(x, p)$, for the case $\gamma=0$. 
There is no conflict here with the uncertainty principle limitation on the idea of a distribution in both $x$ and $p$ simultaneously: the huge spreading out of the coordinate-space distribution eliminates any such conflict.

But what is the rate of approach to this limit?

The probability distribution in coordinate space at $t=0$ for the function in (4.2), at $\gamma=0$, would be given by the (absolute value squared of the) Fourier transform of $\chi(p)$. This transform of the infinitely differentiable compactly supported $\chi(p)$ falls off faster than any power of $|x|$. This leads to the quantum mechanical prediction (4.1). Classically, this original $x$-space distribution is the constant (non-expanding) background to the $t$-dependent diverging trajectories. If this non-expanding background falls off faster than any power of $x$ then its contribution at points $x=u \tau$ will fall off faster than any power of $\tau$. Hence the approach to the large- $t$ limit computed classically, by using the straightline trajectories in space-time, also exhibits the faster than any power fall off specified in (4.1): the classical and quantum predictions agree about both the limit and the rate of approach to this limit.

But what is the rate of fall off for the case $\gamma>0$ ?

To show that the fall off in this case conforms to (4.3) it is sufficient to go to the frame where $\bar{p}$ is pure spacelike and the space part of $u$ is nonzero. Then

$$
\left|\widetilde{\Psi}_{\tau, \gamma, \bar{p}}(u \tau)\right|=\left|\int d^{3} q /(2 \pi)^{-3} \chi(q) \exp \left(-\tau\left[q^{2} \gamma+i\left(q u-u_{0}\left(q_{0}-\bar{p}_{0}\right)\right)\right]\right)\right|
$$

where I again use the metric $(1,1,1)$ for the 3 -vector products $q u$ and $q^{2}$, and $q_{0}-\bar{p}_{0}=\left(q^{2}+m^{2}\right)^{1 / 2}-m$.

To get the quantum prediction, consider a distortion of the $q$-space contour that is parameterized by a scalar $\alpha$. For $q^{2}>\alpha$ there is no distortion. For $R e$ $q^{2}<\alpha$ the component of $\operatorname{Im} q$ that is directed along $u$ is shifted (keeping real the other two components of the 3 -vector $q$ ) so that

$$
\operatorname{Re}\left[q^{2} \gamma+i\left(q u-u_{0}\left(q_{0}-\bar{p}_{0}\right)\right)\right]=\alpha \gamma .
$$

Distort the contour from $\alpha=0$ to a value such that all real $q$ in $q^{2} \leq \alpha$ lie inside the open set where $\chi$ is one, and such that $|\operatorname{Im} q|$ remains less than $m$.

Then for all real points $q$ with $q^{2}>\alpha$ one has an exponential fall-off factor $\exp -\alpha \gamma \tau$. For real $q$ such that $q^{2}<\alpha$ the condition (9.4) gives a factor $\exp -\alpha \gamma \tau$. One can obtain a bound like this for every four vector $u$ on the unit (Euclidean) sphere, minus small open holes around the rays along the positive and negative time axis (along which $\bar{p}$ has been taken to lie). These holes can be defined by conditions on the three-vector part $\vec{u}$ of $u$ : 
$|\vec{u}|<\epsilon$. The only singularity that could block this continuation is the singularity of $q_{0}$ at $q^{2}+m^{2}=0$, and this is prevented by our condition $\mid$ Im $q \mid<m$.

A more detailed presentation is given in Appendix III of Ref. 9.

The classical analog is obtained by taking the classical coordinate-space probability function, imagined now to specify the distribution of the classical particles, to be the one obtained from the Fourier transform. For large $\tau$ the contributions from $\chi-1$ fall off exponentially. Ignoring that contribution, at very large $\tau$, one has a coordinate-space function that is essentially a Gaussian, which has a width that grows like the square root of $\tau$. Hence in the scaleddown coordinate $u=x / \tau$ the width of the Gaussian shrinks like $(\tau)^{-1 / 2}$, just as it does in momentum space. Thus the probability function in $(u, p)$-space (or in $(\vec{u}, \vec{p})$-space) for fixed $(u, p)$, falls off exponentially in $\tau$, as long as one keeps $|\vec{u}|$ finitely away from zero.

The fall-off properties (4.1) and (4.3) pertain to the individual freely moving particles. But we need analogous fall-off properties for process involving multiple scatterings of such freely moving particles by quasi-local interactions.

In quantum theory one has an initial $\Psi_{i n}$ and a final $\Psi_{f i n}$. If a certain preparation procedure $I n$ prepares a system to be in the initial state $\Psi_{i n}$, and if a certain measurement procedure Fin will definitely produce a "Yes" outcome if the final state is $\Psi_{\text {fin }}$, and will definitely produce a "No" outcome if the final state is orthogonal to $\Psi_{\text {fin }}$, then

$$
\text { Probability }=\left|\Psi_{i n}^{*} S \Psi_{f i n}\right|^{2}
$$

is the predicted probability that a preparation of type In followed by a measurement of type Fin will yield an outcome "Yes".

If the intersection of the supports of the wave functions (4.2) contain no points $K$ such that $C(K)$ is bigger than $C_{0}(K)$ then the only relevant classical scattering diagrams are the trivial one that have only one vertex. If the interactions not carried by physical particles have finite range (with perhaps exponential tails) then the transition probability will (as mentioned previously) be bounded, in classical physics, by the probability that all of the particles can be in some region that grows like the square root of $\tau$. And the condition that $C(K)=C_{0}(K)$ for all points in the support of the wave functions means that for any such growing region in spacetime the probability that all the particles will be in this region will have an exponential in $\tau$ fall off coming from some nonzero displacement in either a momentum variable $q$ or a translation variable $u$. And the range of these displacements is compact: they cover the compact surface in $v$ space times the product of the compact domains in $q$-space. Thus 
for these "trivial" points one gets, in the classical-physics analog, a fall off of type (4.3), as already noted.

But how does one get the analogous result for multiple-scattering processes, which involve intermediate particles?

The answer is that if all interaction regions can be taken to grow no faster than the square root of $\tau$, then in the scaled-down (by a factor $\tau$ ) coordinate system the diagram must have point vertices. And momentum-energy is strictly conserved in classical mechanics. So the scaled-down diagrams depict classical processes with point vertices. If no such diagram can match the external conditions imposed by the $(U, K)$ then there will always be an exponential fall-off factor coming from some external particle, which is what the arguments require.

\section{$\S 10$. Nature of the Singularity}

The correspondence principle entails analyticity except on the surfaces specified by the Landau-Nakanishi equations, and it assures analyticity in the associated cones of analyticity at the Landau-Nakanishi points. But what about the nature of these singularities?

Consider a 3-particle to 3-particle process in which two particles collide to create one final particle plus one intermediate particle that eventually collides with the third initial particle to produce the other two final particles. Classical physics demands that in the positive-time asymptotic regime the transition probability function must fall off as $\tau^{-3}$, due to the geometric spreading. This is just the fall off obtained in section 2, and it corresponds to a pole singularity,

$$
f(p)=i\left(p^{2}-m^{2}+i \epsilon\right)^{-1},
$$

which is the energy-increases-with-time part of the mass-shell delta function $2 \pi \delta\left(p^{2}-m^{2}\right)$ of classical physics. Thus not only the location of this singularity, and the $i \epsilon$ rule for continuing around it, but also the pole character of this singularity is determined essentially by the fall-off properties entailed by the correspondence principle.

The geometric conditions that lead to the $\tau^{-3 / 2}$ fall off in the singleintermediate-particle case can be generalized to the case of any number of intermediate particles. One obtains the condition

$$
2 d=3 N_{l}-4\left(N_{v}-1\right)-1,
$$

or

$$
d=\frac{1}{2}\left(3 N_{l}-4 N_{v}+3\right),
$$


where $N_{v}$ is the number of vertices, $N_{l}$ is the number of internal lines, and $d$ is the "degree" of the singularity, with $d=-1$ being $\delta(E)$ or $E^{-1}$, and $d=0$ being $\log E$, etc. Thus for the two-vertex, one internal line case one gets $d=-1$ (a pole singularity) and for the triangle diagram with three vertices and three internal lines one gets $d=0$ (a logarithmic singularity.) For $N_{v}=2$ and $N_{l}=2$ (two-particle threshold) one gets $d=1 / 2,(\sqrt{E})$.

To understand (10.2) from the classical point of view consider the application of (9.1), applied to the entire classical diagram $D$, consisting of $N_{l}$ internal lines, $N_{e}$ external lines, and $N_{v}$ vertices. The factors $\left|\rho\left(u_{i} m_{i}\right) / f\left(m_{i}, t\right)^{2}\right|$, with $t p_{i} / m_{i}=\tau u_{i}$, give the $3 N_{l}$ in (10.2a). Each internal lines contributes a factor $\tau^{-3}$ to the fall-off of the probability, and hence a fall-off factor $\tau^{-3 / 2}$ in the amplitude, and this translates via the Fourier connection to an increase by $3 N_{l} / 2$ of the degree $d$ of the singularity.

But the classical formula (9.1) has also a momentum factor $\rho(p)$. The $p_{i}$ in (9.1) must include an external momentum-energy four-vector at each external line, and the function $\rho(p)$, with $p$ being the collection of internal and external four vectors, will have a conservation-law delta function at each of the $N_{v}$ vertices. This is a classical condition. The scattering function has the one overall conservation-law delta function factored off, leaving $4\left(N_{v}-1\right)$ delta functions.

The term of zeroth order in $N_{l}$ and $N_{v}$ is not determined by this argument, but is fixed by the known pole case to be the extra term -1 in (10.2a). The important point is that to the extent that (10.2) determines the degree $d$ of the singularity, this degree is fixed by the fall-off and conservation-law features exhibited by the associated classical process: the classical process exhibits the features that enter into Eqn. (10.2).

These remarks tie Eqn. (10.2) to classical physics, but do not give a derivation of (10.2). This equation is derived in Kawai and Stapp [12], for all of the cases mentioned above, and, more generally, for each physical-region singularity that corresponds to a unique Landau-Nakanishi diagram in which no two vertices coincide, at most two lines connect any pair of vertices, and no vertex is trivial in the sense that all of the lines connected to it are parallel. [Actually, far more is derived in ref. 12, namely an explicit form of the S-matrix near certain points where several surfaces intersect, and these forms play an important role in understanding the global analytic structure of the S matrix.] The proof is based on the analyticity properties derived from the correspondences principle, on the general theory of holonomic microfunctions described in Sato, Kawai, and Kashiwara [13], and on the techniques and results developed in Coster and Stapp $[14,15]$ for combining the analytity properties that follow 
from the correspondence principles with the important unitarity property of the $\mathrm{S}$ matrix.

The other key element in S-matrix theory is "crossing": the postulate that a certain analytic continuation that changes $k_{i}$ to $-k_{i}$ will take one to the scattering function of a "crossed" process where initial (resp. final) particle $i$ is replaced by final (resp. initial) anti-particle $i$. Hence much of the structure of quantum theory is seen to be entailed already by the correspondence principle, plus natural extensions of the analyticity properties entailed by the correspondence principle.

\section{§11. Photons and Infra-Red Divergences}

Massless particles, such as photons, pose new technical problems, which are entwined with an important infra-red problem. A number of studies $[16,17,18]$ of the effects of the interaction of an electron (or positron) with low-energy photons appeared to show that the pole-character of the electron is disrupted by this interaction: the pole exponent -1 is modified by a term of order $1 / 137$. However, any such change at the level of the $\mathrm{S}$ matrix itself would entail a significant deviation from the $1 / r^{3}$ fall off, which is empirically confirmed to very high accuracy.

Part of the problem in those works is that what was studied was the electron propagator, which corresponds, physically, to suddenly creating a charged electron at some point $x$ and suddenly destroying it at some other point $x^{\prime}$. But charge is conserved: it cannot be suddenly created or destroyed. So one should examine, instead, closed loops of charge, where two particles of opposite charge emerge from an initial place, and eventually come together at some later place. But even when this is done there still remains an infra-red divergence problem, associated with the emission of "infinite" numbers of soft (i.e., low-energy) photons at each place where some deflection or deviation of the spacetime trajectory of the charged particle from straight-line motion occurs. This infra-red problem is solved by again appealing to the correspondence principle.

The point is this. If one considers the space-time diagram associated with the Feynman graph as a classical multiple-scattering process - of charged particles - then one can compute the classical electromagnetic field radiated by those moving charges. It has long been known that for every classical radiation field there is a corresponding quantum state, called a coherent state. It involves infinite numbers of photons. To resolve the infra-red divergence problem completely one should use for the final quantum state of the radiant electromagnetic field, not the vacuum state plus added photons, but rather the quantum co- 
herent state corresponding to the classical electromagnetic field radiated from the classical process specified by the Landau-Nakanishi diagram, plus added photons. So again, as before, the quantum process is largely determined by the underlying classical process: the classical process determines the bulk of the radiated quantum electromagnetic field, and once this part is properly incorporated the fall-off properties associated with motions of the charged particles come into proper accord with the predictions of classical physics, which then fixes, via analyticty, the parts of the quantum scattering function closely associated with this classical process. One can then, again, reverse engineer the correspondence principle to get the quantum counterpart of the classical process. The program was initiated by Stapp [19], and various resulting analyticity properties were derived in a series of papers by Kawai and Stapp [20, 21].

In the works described above the particle trajectories were always taken to be straight-line segments. However, Eqn. (2.16) of ref. 19 shows the effect of the "Coulomb" contribution. It conforms to the classical rule. The correspondence principle approach discussed here suggests allowing the classical-particle trajectory to deviate from straight lines in a way that gives stationary action. That will cause these classical trajectories to curve as they do classically under the influence of a Coulomb potential. These curved trajectories will radiate soft photons that will need to be added to the final coherent state.

This suggested application of the correspondence principle begins to look more like a traditional spacetime description than an S-matrix calculation. However, it is built not upon the presumption of local interactions but rather upon analyticity properties derived by a reverse engineering of the correspondence-principle classical limit.

\section{References}

[1] Wheeler, J. A., On the mathematical description of light nuclei by the method of resonating group structure, Phys. Rev., 52 (1937), 1107-1122.

[2] Heisenberg, W., Die "beobachtbaren grossen" in der theorie der elementarteilen I and II, Z. Physik, 120 (1943), 513-538, ibid. 673-702.

[3] Heisenberg, W., ref. 2; Chew, G. F., S-Matrix Theory of Strong Interactions, W.A. Benjamin, New York, 1961; G. F.Chew, The Analytic S-Matrix, W.A. Benjamin, New York, 1966.

[4] Landau, L. D., On analytic properties of vertex parts, Nucl. Phys., 13 (1959), 181-192.

[5] Nakanishi, N., Ordinary and anomalous thresholds in perturbation theory, Prog. Theor. Phys., 22 (1959), 128-144.

[6] Coleman, S. and Norton, R., Singularities in the physical region, Nuovo Cimento, 38 (1965), 438-442.

[7] Chandler, C. and Stapp, H., Macroscopic causality conditions and properties of scattering functions, J. Math. Phys., 10 (1969), 826-859. 
[8] Iagolnitzer, D. and Stapp, H., Macroscopic causality and physical region analyticity in S-matrix theory, Comm. Math. Phys., 14 (1969), 15-55.

[9] Sato, M., Hyperfunctions and partial differential equations, Proc. Internat. Conf. on Functional Analysis and Related Topics, 1969, pp. 91-94, Univ. Tokyo Press. Tokyo, 1970.

[10] Stapp, H., Finiteness of the number of positive-alpha Landau singularity surfaces in bounded portions of the physical region, J. Math. Phys., 8, (1967), 1606-1610.

[11] Iagonitzer, D., The S Matrix. North-Holland, New York, 1976.

[12] Kawai, T. and Stapp, H., Discontinuity formula and Sato's Conjecture, Publ. RIMS, Kyoto Univ., 12 Suppl. (1977), 155-232.

[13] Sato, M., Kawai, T. and Kashiwara, M., Microfunctions and pseudo- differential equations, Lecture Notes in Math., 287, pp. 265-529, Berlin-Heidelberg-New York, Springer, 1973.

[14] Coster, J. and Stapp, H., Physical region discontinuity equations, J. Math. Phys., 11 (1970), 2743-2763.

[15] I, J. Math. Phys., 10 (1969), 371-396.

[16] Kibble, T., Coherent soft-photon states and infrared divergences IV, Phys. Rev., 175 (1968), 1624-1640.

[17] Zwanziger, D., Reduction formulas for charged particles and coherent states in quantum electrodynamics, Phys. Rev., D7 (1973), 1062-1098.

[18] Storrow, J. K., Photons in S-matrix theory, Nuovo Cimento, 54A (1968), 15-41, and 57A (1968), 763-776.

[19] Stapp, H. P., Exact solution of the infrared problem, Phys. Rev., D28 (1983), 1386-1418.

[20] Kawai, T. and Stapp, H., Quantum electrodynamics at large distances, Phys. Rev., D52 (1995), 2484-2532.

[21] On infra-red singularities associated with QC photons, Microlocal Analysis and Complex Fourier Analysis, World Scientific Publishing, Singapore, 2002, pp. 115-134. 